\title{
Nonstandard FDTD Simulation-Based Design of CROW Wavelength Splitters
}

\author{
Naoki Okada, ${ }^{1}$ James B. Cole, ${ }^{1}$ Shigeki Yamada, ${ }^{2}$ Kensuke Ogawa, $^{3}$ and Yoshifumi Katayama ${ }^{4}$ \\ ${ }^{1}$ Department of Computer Science, Graduate School of Systems and Information Engineering, University of Tsukuba, 1-1-1 Tennodai, \\ Tsukuba, Ibaraki 305-8573, Japan \\ ${ }^{2}$ Department of Nanosystem Science, Graduate School of Nanobioscience, Yokohama City University, 22-2 Seto, Kanazawa-ku, \\ Yokohama, Kanagawa 236-0027, Japan \\ ${ }^{3}$ Optics and Electronics Laboratory, Fujikura Ltd., 1440 Mutsuzaki, Sakura, Chiba 285-8550, Japan \\ ${ }^{4}$ Koga Soken Ltd., 344-6 Uchikoshi-machi, Hachioji, Tokyo 192-0082, Japan
}

Correspondence should be addressed to Naoki Okada, okada@cavelab.cs.tsukuba.ac.jp

Received 27 January 2011; Accepted 23 March 2011

Academic Editor: Augusto Belendez

Copyright (C) 2011 Naoki Okada et al. This is an open access article distributed under the Creative Commons Attribution License, which permits unrestricted use, distribution, and reproduction in any medium, provided the original work is properly cited.

\begin{abstract}
The finite-difference time-domain (FDTD) algorithm has been used in simulation-based designs of many optical devices, but it fails to reproduce high-Q whispering gallery modes (WGMs). On the other hand, the nonstandard (NS) FDTD algorithm can accurately compute WGMs and can be used to make simulation-based designs of WGM devices. Wavelength splitters using the coupled resonator optical waveguides (CROWs) based on WGM couplings have recently attracted attention because they are potentially ultracompact. In this paper, we design a CROW wavelength splitter using NS FDTD simulations and demonstrate high interchannel extinction ratios of over $20 \mathrm{~dB}$.
\end{abstract}

\section{Introduction}

The finite-difference time-domain (FDTD) algorithm [1] has been used in simulation-based designs of optical devices such as optical fibers. However, the FDTD algorithm has not been used to design optical disc and ring resonators based on whispering gallery modes (WGMs) in the past, although these are essential building blocks of integrated optical circuits. This is due to failure of the conventional FDTD algorithm to reproduce the high-Q WGM resonances [2-4]. Instead the discontinuous Galerkin time-domain (DGTD) method [5] which can accurately calculate these resonances with lower memory consumption has been used, but its computational overhead is significantly higher than FDTD [6]. Recently, the nonstandard (NS) FDTD algorithm $[7,8]$ has been successfully used for high-accuracy WGM simulations with lower memory consumption and computational overhead $[9,10]$. This is because high-accuracy difference operators are derived by optimizing to monochromatic wave propagation in the NS-FDTD algorithm and its temporal-spatial difference errors are considerably reduced by comparison with the conventional FDTD. Details of the NS-FDTD derivation are given in $[8,9]$. Thus, the NS-FDTD algorithm can be used to make simulation-based designs of WGM devices.

On the other hand, the coupled resonator optical waveguide (CROW) $[11,12]$ has attracted much attention in recent years because it combines characteristics of both resonator and waveguide and is potentially ultracompact. In particular, the optical wavelength splitter using the CROW $[13,14]$ is very small in comparison with conventional splitters. For example, the microring resonator-based splitter [15] has high interchannel extinction ratios (IERs), but its size is much larger than a wavelength (a few dozen $\mu \mathrm{m}$ ) because the resonator employs total internal reflection with designs based on geometric optics theory. Other splitters using an arrayed waveguide grating [16] and multimode interferometer [17] are also large and have just barely enough IERs because they are not resonators. In contrast, the CROW splitter has high IERs and is potentially ultracompact (on the order of a wavelength) because the CROW is based on WGM couplings between contiguous disk resonators in the Mie regime. 


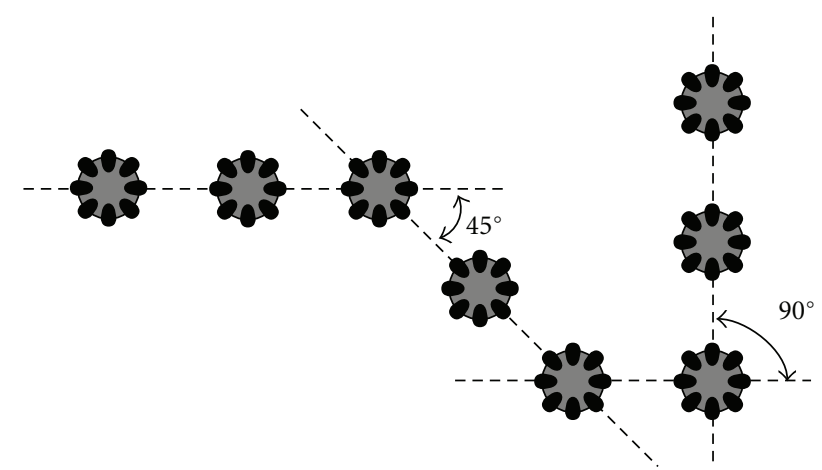

FIgUre 1: Flexible layout in the CROW. The gray regions represent microdisks, and black regions show whispering gallery modes in each disk.

In this paper, we design two CROW wavelength splitters based on the NS-FDTD simulation and demonstrate its high IERs of over $20 \mathrm{~dB}$.

\section{Coupled Resonator Optical Waveguide}

The coupled resonator optical waveguide (CROW) consisting of microdisks propagates energy with low loss by coupling whispering gallery mode (WGM) resonators in the Mie regime. As shown in Figure 1, the microdisks can be arranged to form any path. Thus, we can flexibly design optical devices.

The resonance wavelength and disk size are calculated using the WGM resonance condition. The resonance condition in geometric optics theory is given by [18]

$$
2 \pi r=\ell \frac{\lambda}{n} \quad\left(r \gg \frac{\lambda}{n}\right),
$$

where $r$ is the disk radius, $\lambda$ is the wavelength outside the disk, $n$ is the specific refractive index of the disk, and $\ell$ is the mode number (integer). These high mode WGMs are widely used in many optical devices such as microring resonators, but they are much larger than a wavelength because high mode resonators employ total internal reflection.

On the other hand, in the Mie regime, the size of WGM resonators is of the order of a wavelength and the resonance conditions are given by the Mie theory $[19,20]$. In the transverse electric (TE) mode in which the electric field is perpendicular on the media interfaces, the resonance condition is

$$
\frac{J_{\ell-2}(n k r)}{J_{\ell-1}(n k r)}=n \frac{H_{\ell-2}^{(1)}(k r)}{H_{\ell-1}^{(1)}(k r)}-\frac{(\ell-1)\left(n^{2}-1\right)}{n k r},
$$

where $k=2 \pi / \lambda, J_{\ell}$ is the Bessel function of the first kind, and $H_{\ell}^{(1)}$ is the Hankel function of the first kind. In the transverse magnetic (TM) mode in which the electric field is parallel to the media interfaces, the resonance condition is

$$
\frac{J_{\ell-1}(n k r)}{J_{\ell}(n k r)}=\frac{1}{n} \frac{H_{\ell-1}^{(1)}(k r)}{H_{\ell}^{(1)}(k r)} .
$$

We can numerically compute the appropriate disk size for a given wavelength and refractive index using (2) and (3).
TABLE 1: Example resonance conditions. The specific refractive index between $\mathrm{Si}$ and $\mathrm{SiO}_{2}$ is $n=3.5 / 1.45$.

\begin{tabular}{lcc}
\hline & TE mode & \\
\hline Wavelength $\lambda$ & Radius $r$ & Mode $\ell$ \\
\hline $1350 \mathrm{~nm}$ & $953.72 \mathrm{~nm}$ & 8 \\
$1400 \mathrm{~nm}$ & $1096.58 \mathrm{~nm}$ & 9 \\
$1450 \mathrm{~nm}$ & $1245.55 \mathrm{~nm}$ & 10 \\
$1500 \mathrm{~nm}$ & $1400.82 \mathrm{~nm}$ & 11 \\
$1550 \mathrm{~nm}$ & $1562.53 \mathrm{~nm}$ & 12 \\
\hline & TM mode & \\
\hline Wavelength $\lambda$ & Radius $r$ & 5 \\
$1350 \mathrm{~nm}$ & $655.02 \mathrm{~nm}$ & 6 \\
$1400 \mathrm{~nm}$ & $790.51 \mathrm{~nm}$ & 7 \\
$1450 \mathrm{~nm}$ & $931.76 \mathrm{~nm}$ & 8 \\
$1500 \mathrm{~nm}$ & $1078.97 \mathrm{~nm}$ & 9 \\
$1550 \mathrm{~nm}$ & $1232.32 \mathrm{~nm}$ & \\
\hline
\end{tabular}

Example resonance conditions are listed in Table 1, where we choose the widely used specific refractive index between silicon $(\mathrm{Si})$ inside the disk and silicon dioxide $\left(\mathrm{SiO}_{2}\right)$ outside.

\section{Optical Wavelength Splitter}

In the CROW, disks of different size (tuned to specific wavelengths) can be used to select the components of a multiplexed input signal and function as a wavelength splitter. The interchannel extinction ratio is high because the disk resonators are strongly excited and coupled only at specific wavelengths. This CROW splitter is much smaller than conventional devices since the resonator sizes are comparable to a wavelength. Moreover, the CROW splitter can be used in multiplex polarization communication because the resonance conditions of (2) and (3) are polarization dependent unlike conventional devices.

We design two CROW splitters. The first device separates wavelength components from a disk resonator which is concurrently excited by two different wavelengths as shown in Figure 2. The WGMs of wavelength $\lambda_{A}$ and $\lambda_{B}$ are excited in a disk $O$. Disks $A$ and $B$ are excitable only by $\lambda_{A}$ and $\lambda_{B}$, respectively. We empirically computed the appropriate gap spacings between disks, $\Delta=0.2 r$ ( $r=$ disk radius). If $\Delta$ is too small, both the desired wavelength and undesired one propagate. But if $\Delta$ is too large, the desired wavelength is also suppressed.

The second device extracts arbitrary wavelengths from an optical waveguide as shown in Figure 3. A multiplexed input signal of wavelengths $\lambda_{A}$ and $\lambda_{B}$ propagates in the optical waveguide. Disks $A$ and $B$ are excitable only by $\lambda_{A}$ and $\lambda_{B}$, respectively.

\section{Simulation and Validation}

We calculated the electromagnetic wave propagation in two devices (Figures 2 and 3 ) using the nonstandard (NS) finitedifference time-domain (FDTD) algorithm in the TE mode and demonstrated the interchannel extinction ratios (IERs) 


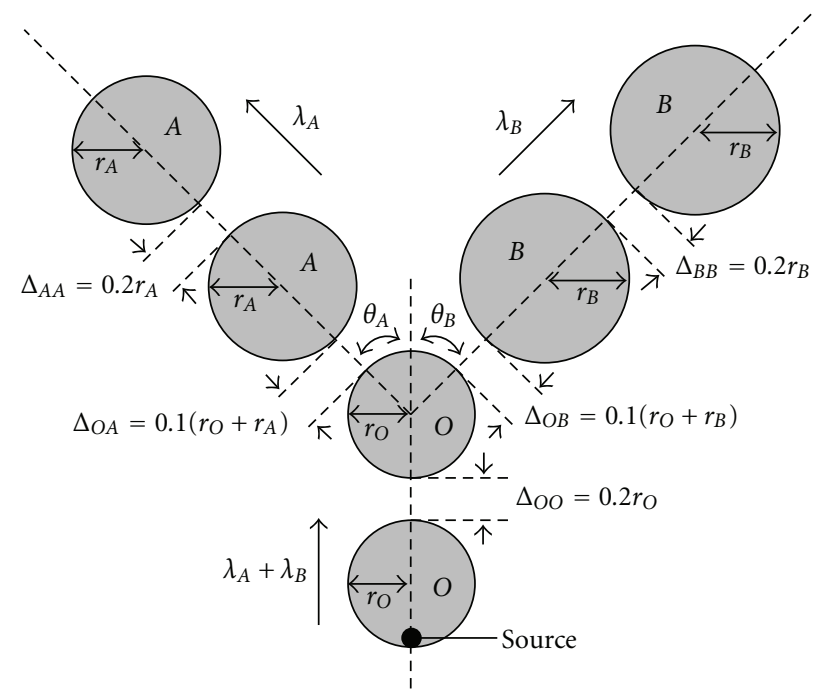

FIGURE 2: Wavelength splitter for disk resonators. Gray regions represent microdisks with excited WGMs. $r_{O}, r_{A}$, and $r_{B}$ are the radii of disks $O, A, B$, respectively. $\Delta_{O A}$ is the gap spacing between disks $O$ and $A$ ( $\Delta_{O O}, \Delta_{O B}$, etc., analogously defined). Source is a superposition of wavelengths $\lambda_{A}$ and $\lambda_{B}$. WGMs of $\lambda_{A}$ and $\lambda_{B}$ are excited in disks $O$. Disks $A$ and $B$ are excitable only by $\lambda_{A}$ and $\lambda_{B}$, respectively.

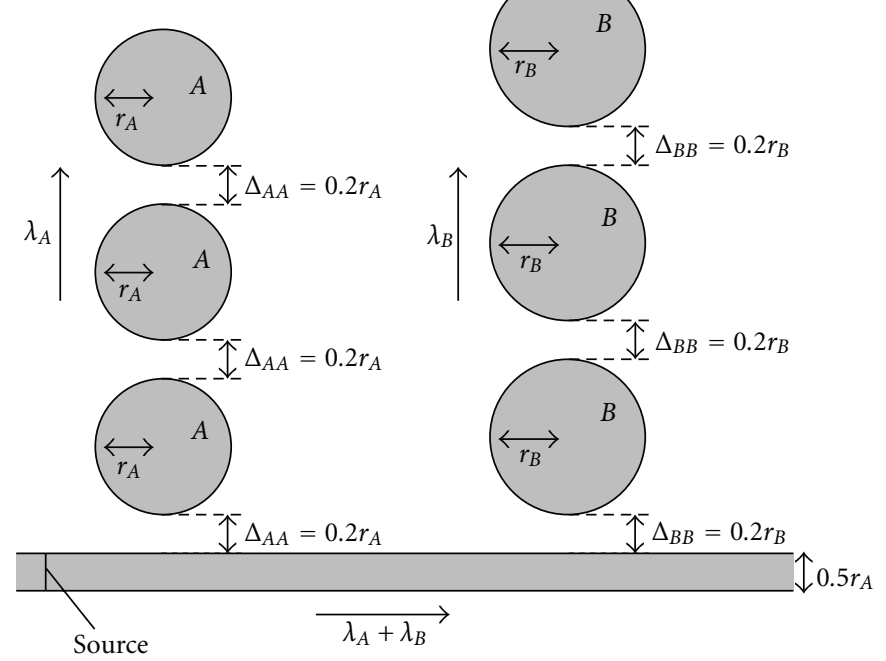

FIGURE 3: Wavelength splitter for multimode signals in an optical waveguide. Circular gray regions represent microdisks with excited WGMs. Linear gray regions represent an optical waveguide. $r_{A}$ and $r_{B}$ are the radii of disks $A$ and $B$, respectively. $\Delta_{A A}$ is the gap spacing between disks $A$ ( $\Delta_{B B}$ analogously defined). Source is a superposition of wavelengths $\lambda_{A}$ and $\lambda_{B}$. Disks $A$ and $B$ are excitable only by $\lambda_{A}$ and $\lambda_{B}$, respectively.

and effects of manufacturing errors. The computational domain is terminated by the NS perfectly matched layer absorbing boundary condition [21]. In the TM mode, similar devices and functions can be computed.

First we demonstrated that whispering gallery modes (WGMs) are accurately simulated by the NS-FDTD algorithm. Example parameters are listed in Table 2. In the TE mode, we calculated the scattered intensity of the electric field due to an infinite plane wave using the NS-FDTD algorithm and compared the result with the Mie theory in Figure 4. Figures 4(a) and 4(b) visualize scattered intensity distributions of analytical and numerical results at steady state (about 1000 wave periods). Figure 4(c) shows angular scattered intensity distributions on a circular contour of radius $1.1 r(r=$ disk radius $)$ around the disk center. As shown in Figure 4, the NS-FDTD algorithm accurately computes the WGM.

Before simulating the splitter shown in Figure 2, we must select the point source position because resonance intensity is sensitive to source position. We simulated WGMs in a disk using different source positions with parameters listed in Table 2. Figure 5 shows the average total intensity over the surface of a disk at steady state (about 2000 wave periods) for different source positions. As shown in Figure 5, if the source position is too far from the surface or too close, waves cross the surface are radiated outside the disk. For the 8 mode 


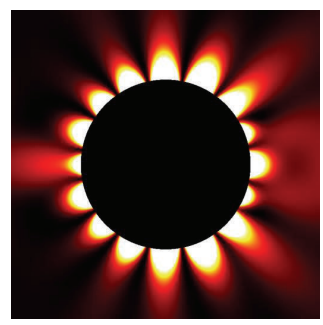

(a)

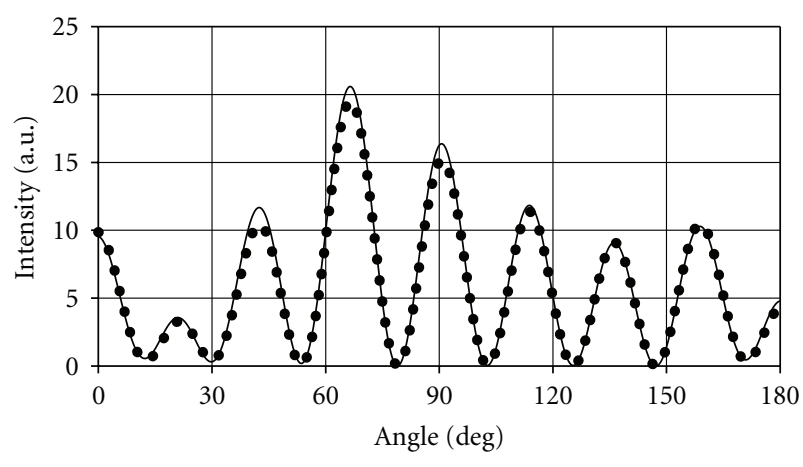

- Analytical

- - Numerical

(c)

FIgURE 4: Scattered intensities of electric fields at steady state, TE mode. (a) Analytical solution. (b) Numerical result by the NSFDTD algorithm. (c) Angular intensity distributions of (a) and (b) on a circular contour of radius $1.1 r$ ( $r=$ disk radius) around the disk center.

TABLE 2: Example parameters used to simulate $\ell=8$ mode WGM in the TE mode.

\begin{tabular}{lc}
\hline Wavelength & $1550 \mathrm{~nm}$ \\
Disk radius & $1095 \mathrm{~nm}$ \\
Grid spacing & $5 \mathrm{~nm}$ \\
Refractive index & $3.5 / 1.45\left(\mathrm{Si}^{\prime} / \mathrm{SiO}_{2}\right)$ \\
Computational space & $4 \mu \mathrm{m} \times 4 \mu \mathrm{m}$ \\
\hline
\end{tabular}

resonance in Figure 5, the strongest resonance is obtained at source position $=0.75 r$, but the optimal source position depends on the resonance mode. In low modes, we found that the optimal source position is shifted from $0.7 r$ (at 6 mode) to $0.8 r$ (at 12 mode). In the following simulations, we chose source position $=0.775 r$ because both $\ell=9$ and $\ell=11$ modes are excited in disk $O$ (Figure 2).

We simulated the device shown in Figure 2 for $2^{20}$ time steps (about 2400 wave periods) and observed the IER on ports set at resonance-enhanced points outside disks $A$ and $B$. We gradually damp the source amplitude after $2^{19}$ time steps to reduce spurious frequency components in the Fourier transform. We used the simulation parameters listed in Table 3. These wavelengths are widely used in optical communication. Figures 6(a) and 6(b) show the computed spectra on ports $A$ and $B$, respectively. As shown in Figure 6 , both

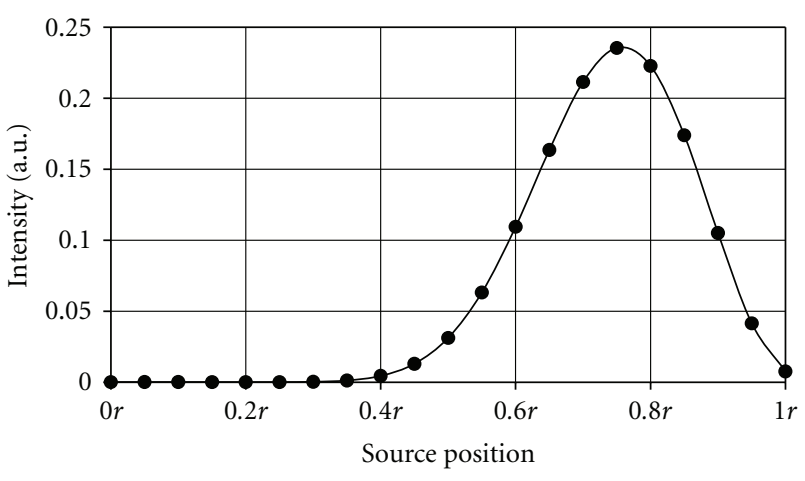

FIGURE 5: Effect of source positions on an $L=8$ WGM resonance intensity. The source position is shifted from the center $=0.0 r$ to the surface $=1.0 r$ ( $r=$ disk radius $)$. The intensity is an average over the surface of a disk at steady state.

TABle 3: Computational parameters and resonance modes for the wavelength splitter shown in Figure 2. $\theta_{A}$ and $\theta_{B}$ are integral multiples of $\pi / \ell \operatorname{rad}(\ell=$ mode number in the disk $O)$.

\begin{tabular}{lcc}
\hline \multicolumn{3}{c}{ Computational parameters } \\
\hline$\lambda_{A}$ & $1300 \mathrm{~nm}$ \\
$\lambda_{B}$ & $1550 \mathrm{~nm}$ \\
$r_{O}$ & $1214.08 \mathrm{~nm}$ \\
$r_{A}$ & $1310.52 \mathrm{~nm}$ \\
$r_{B}$ & $1562.54 \mathrm{~nm}$ \\
$\theta_{A}$ & $3 \pi / 11 \mathrm{rad}$ \\
$\theta_{B}$ & $3 \pi / 9 \mathrm{rad}$ \\
Grid spacing & $5 \mathrm{~nm}$ \\
Refractive index & $3.5 / 1.45\left(\mathrm{Si} / \mathrm{SiO} \mathrm{O}_{2}\right)$ \\
Computational space & $14 \mu \mathrm{m} \times 12 \mathrm{~m}$ \\
\hline \multicolumn{4}{c}{ Resonance modes } \\
\hline Port & $\lambda_{A}$ & \multicolumn{2}{c}{$\lambda_{B}$} \\
\hline$O$ & 11 mode \\
$A$ & 12 mode & unexcited \\
$B$ & unexcited & 12 mode \\
\hline
\end{tabular}

IERs between $\lambda_{A}=1300 \mathrm{~nm}$ and $\lambda_{B}=1550 \mathrm{~nm}$ are over $20 \mathrm{~dB}(26.3 \mathrm{~dB}$ on port $A ; 29.1 \mathrm{~dB}$ on port $B)$.

We similarly simulated the device shown in Figure 3 for $2^{20}$ time steps (gradually damping the source after $2^{19}$ time steps) and observed IERs on ports set at resonance-enhanced points outside disks $A$ and $B$. We used the simulation parameters listed in Table 4. Figures $7(\mathrm{a})$ and $7(\mathrm{~b})$ show the computed spectra on the port $A$ and $B$, respectively. As shown in Figure 7, both IERs between $\lambda_{A}=1400 \mathrm{~nm}$ and $\lambda_{B}=1550 \mathrm{~nm}$ are over $20 \mathrm{~dB}(21.5 \mathrm{~dB}$ on port $A ; 24.5 \mathrm{~dB}$ on port $B)$.

Finally, we examined effects of manufacturing errors for a practical realization. The radius error $\Delta r$ and refractive index error $\Delta n$ are produced during the manufacturing process and generate the shift of the resonance wavelength $\Delta \lambda$. In 


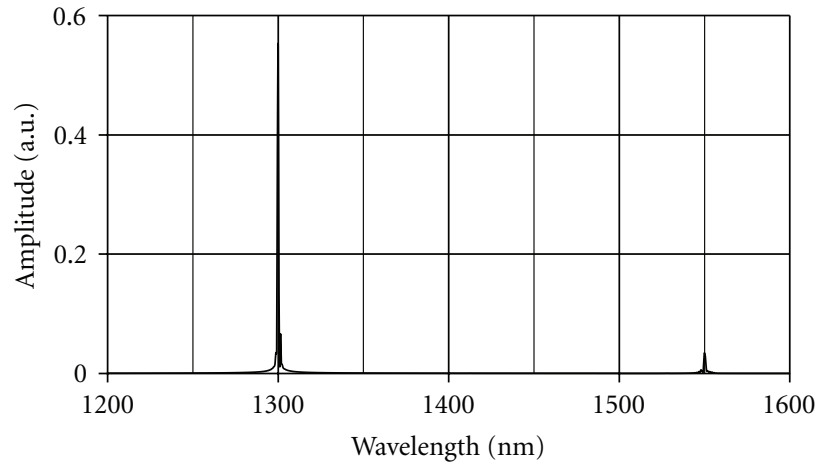

(a)

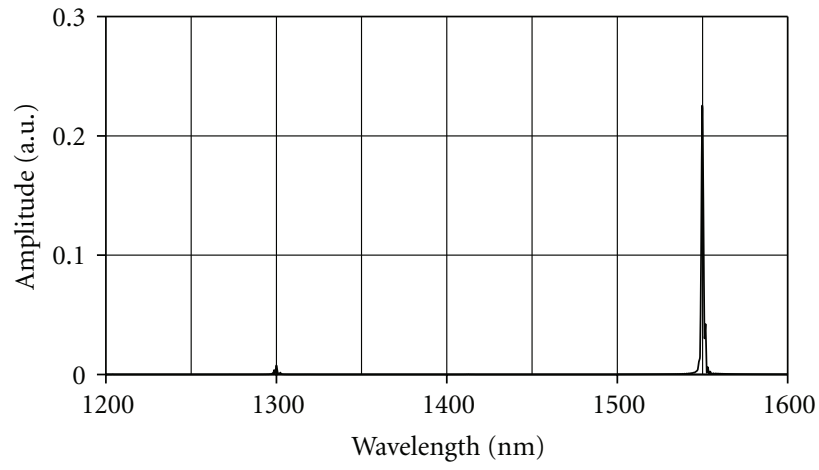

(b)

Figure 6: Wavelength spectra from the simulation of the wavelength splitter of Figure 2. The observation ports are set at resonance-enhanced points outside disks $A$ and $B$. (a) Spectrum on the port $A$. Interchannel extinction ratios $=26.3 \mathrm{~dB}$. (b) Spectrum on the port $B$. Interchannel extinction ratio $=29.1 \mathrm{~dB}$.

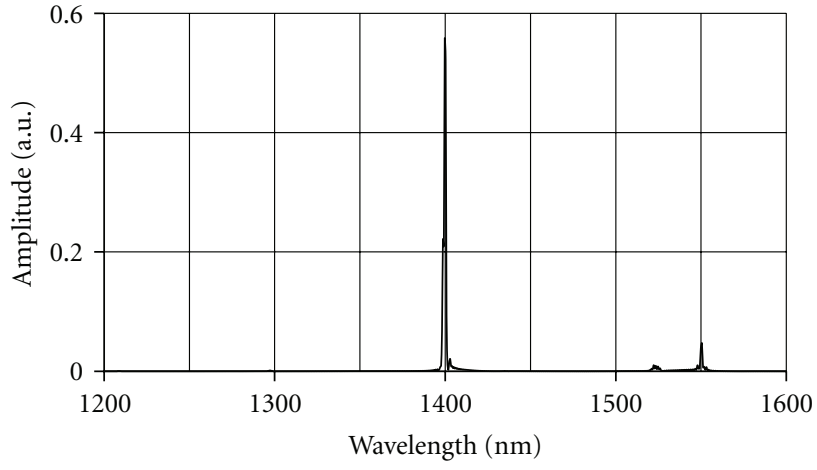

(a)

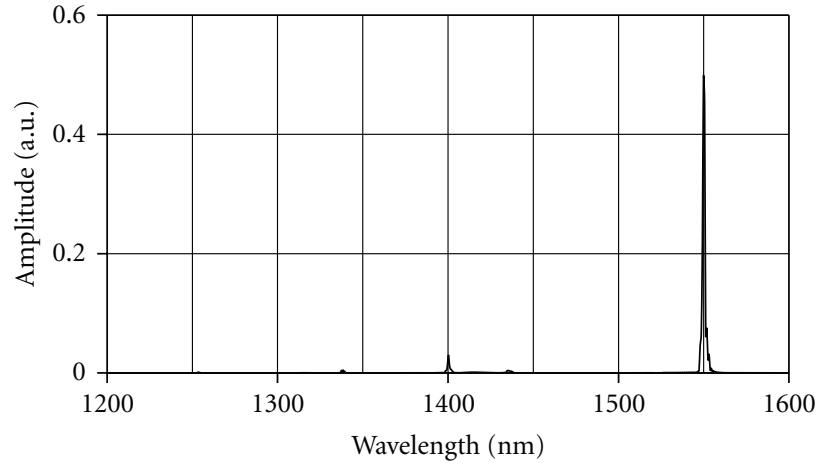

(b)

FIGURE 7: Wavelength spectra from the simulation of the wavelength splitter of Figure 3. The observation ports are set at resonance-enhanced points outside disks $A$ and $B$. (a) Spectrum on port $A$. Interchannel extinction ratio $=21.5 \mathrm{~dB}$. (b) Spectrum on port $B$. Interchannel extinction ratio $=24.5 \mathrm{~dB}$.

TABLE 4: Computational parameters and resonance modes in the wavelength splitter shown in Figure 3.

\begin{tabular}{lcc}
\hline \multicolumn{3}{c}{ Computational parameters } \\
\hline$\lambda_{A}$ & $1400 \mathrm{~nm}$ \\
$\lambda_{B}$ & $1550 \mathrm{~nm}$ \\
$r_{A}$ & $1307.43 \mathrm{~nm}$ \\
$r_{B}$ & $1447.51 \mathrm{~nm}$ \\
Grid spacing & $5 \mathrm{~nm}$ \\
Refractive index & $3.5 / 1.45\left(\mathrm{Si} / \mathrm{SiO}_{2}\right)$ \\
Computational space & $12 \mu \mathrm{m} \times 12 \mu \mathrm{m}$ \\
\hline \multicolumn{4}{c}{$\lambda_{B}$} \\
\hline Port & Resonance modes & Unexcited \\
\hline$A$ & \multicolumn{2}{c}{11 mode } \\
$B$
\end{tabular}

the TE and TM resonance conditions, we found that $\Delta \lambda$ is approximated by

$$
\Delta \lambda \cong \frac{\lambda_{\ell}}{r_{\ell}} \Delta r+\frac{\lambda_{\ell+1}}{n_{\ell}} \Delta n
$$

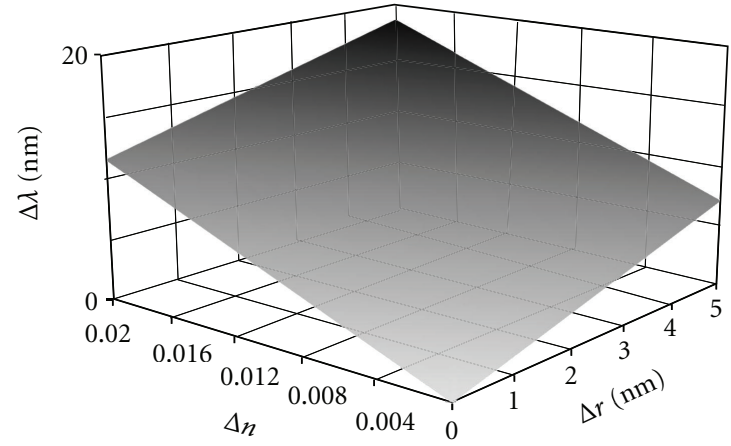

FIGURE 8: Effects of manufacturing errors. $\Delta r$ is the radius error. $\Delta n$ is the refractive index error. $\Delta \lambda$ is the shift of the resonance wavelength.

where $\lambda_{\ell}, r_{\ell}$, and $n_{\ell}$ are the wavelength, radius, and refractive index on the $\ell$ mode resonance $\left(r_{\ell} \rightarrow r_{\ell}+\Delta r, n_{\ell} \rightarrow n_{\ell}+\right.$ $\left.\Delta n \Rightarrow \lambda_{\ell} \rightarrow \lambda_{\ell}+\Delta \lambda\right)$. The relationship between $\Delta \lambda$ and $\Delta r$ is derived from $2 \pi r_{\ell} / \lambda_{\ell}=2 \pi\left(r_{\ell}+\Delta r\right) /\left(\lambda_{\ell}+\Delta \lambda\right)$, where the resonance condition is maintained. The relationship 
between $\Delta \lambda$ and $\Delta n$ is more complex and is numerically approximated. Figure 8 shows the $\Delta \lambda$ distribution using example parameters listed in Table 2 . The latest lithography gives $\Delta r \cong 5 \mathrm{~nm}$ and thus $\Delta \lambda \cong 7 \mathrm{~nm}(\Delta n=0)$ as shown in Figure 8 . The desired resonance wavelength $(\Delta \lambda=0)$ is obtained by adjusting the refractive index of the silicon, which depends on the temperature [22].

\section{Conclusion}

Based on the nonstandard (NS) finite-difference timedomain (FDTD) simulations, we designed two ultracompact optical wavelength splitters using the coupled resonator optical waveguide (CROW) and demonstrated high interchannel extinction ratios (IERs) of over $20 \mathrm{~dB}$. The first device in Figure 2 separates wavelengths from a disk resonator and also can function as a source separator. The second device in Figure 3 extracts arbitrary wavelengths from an optical waveguide and can theoretically split any multimode signal. These splitters can be used in multiplex polarization communication, since low mode whispering gallery modes are polarization dependent. In some applications polarization independence is desirable, but this could be achieved by using two disks excited in each mode.

The NS-FDTD algorithm has much lower computational overhead than the discontinuous Galerkin time-domain method and is much simpler to program. In this paper, we presented the NS-FDTD simulation-based design as a prototype tool for the design of more advanced devices such as photonic-crystal circuits. In future work we will try to optimize the gap spacing and obtain a higher IER, within such technological constraints as semiconductor lithography and wavelength dispersion.

\section{References}

[1] K. S. Yee, "Numerical solution of initial boundary value problems involving maxwell's equations in isotropic media," IEEE Transactions on Antennas and Propagation, vol. 14, no. 3, pp. 302-307, 1966.

[2] G. L. Hower, R. G. Olsen, J. D. Earls, and J. B. Schneider, "Inaccuracies in numerical calculation of scattering near natural frequencies of penetrable objects," IEEE Transactions on Antennas and Propagation, vol. 41, no. 7, pp. 982-986, 1993.

[3] A. Hoekstra, J. Rahola, and P. Sloot, "Accuracy of internal fields in volume integral equation simulations of light scattering," Applied Optics, vol. 37, no. 36, pp. 8482-8497, 1998.

[4] S. V. Boriskina, P. Sewell, and T. M. Benson, "Accurate simulation of two-dimensional optical microcavities with uniquely solvable boundary integral equations and trigonometric galerkin discretization," Journal of the Optical Society of America A, vol. 21, no. 3, pp. 393-402, 2004.

[5] X. Ji, T. Lu, W. Cai, and P. Zhang, "Discontinuous galerkin time domain (DGTD) methods for the study of 2-D waveguide-coupled microring resonators," Journal of Lightwave Technology, vol. 23, no. 11, pp. 3864-3874, 2005.

[6] J. Niegemann, W. Pernice, and K. Busch, "Simulation of optical resonators using DGTD and FDTD," Journal of Optics A, vol. 11, no. 11, Article ID 114015, 2009.
[7] R. E. Mickens, Nonstandard Finite Difference Models of Differential Equation, World Scientific, 1994.

[8] J. B. Cole, "High-accuracy yee algorithm based on nonstandard finite differences: new developments and verifications," IEEE Transactions on Antennas and Propagation, vol. 50, no. 9, pp. 1185-1191, 2002.

[9] N. Okada and J. B. Cole, "Simulation of whispering gallery modes in the mie regime using the nonstandard finitedifference time domain algorithm," Journal of the Optical Society of America B, vol. 27, no. 4, pp. 631-639, 2010.

[10] N. Okada and J. B. Cole, "High-accuracy finite-difference time domain algorithm for the coupled wave equation," Journal of the Optical Society of America B, vol. 27, no. 7, pp. 1409-1413, 2010.

[11] A. Yariv, Y. Xu, R. K. Lee, and A. Scherer, "Coupled-resonator optical waveguide: a proposal and analysis," Optics Letters, vol. 24, no. 11, pp. 711-713, 1999.

[12] S. Deng, W. Cai, and V. N. Astratov, "Numerical study of light propagation via whispering gallery modes in microcylinder coupled resonator optical waveguides," Optics Express, vol. 12, no. 26, pp. 6468-6480, 2004.

[13] S. J. Emelett and R. Soref, "Design and simulation of silicon microring optical routing switches," Journal of Lightwave Technology, vol. 23, no. 4, pp. 1800-1807, 2005.

[14] S. V. Boriskina, "Spectral engineering of bends and branches in microdisk coupled-resonator optical waveguides," Optics Express, vol. 15, no. 25, pp. 17371-17379, 2007.

[15] P. Dumon, W. Bogaerts, D. Van Thourhout et al., "Compact wavelength router based on a Silicon-on-insulator arrayed waveguide grating pigtailed to a fiber array," Optics Express, vol. 14, no. 2, pp. 664-669, 2006.

[16] G. Yuan, P. Wang, Y. Lu, and H. Ming, "Multimode interference splitter based on dielectric-loaded surface plasmon polariton waveguides," Optics Express, vol. 17, no. 15, pp. 12594-12600, 2009.

[17] V. Van, T. A. Ibrahim, K. Ritter et al., "All-optical nonlinear switching in GaAs-AlGaAs microring resonators," IEEE Photonics Technology Letters, vol. 14, no. 1, pp. 74-76, 2002.

[18] F. Vollmer and S. Arnold, "Whispering-gallery-mode biosensing: Label-free detection down to single molecules," Nature Methods, vol. 5, no. 7, pp. 591-596, 2008.

[19] P. W. Barber and S. C. Hill, Light Scattering by Particles: Computational Methods, World Scientific, 1989.

[20] J. Heebner, R. Grover, and T. A. Ibrahim, Optical Microresonators: Theory, Fabrication and Applications, Springer, 2007.

[21] N. Okada and J. B. Cole, "Nonstandard finite difference time domain algorithm for berenger's perfectly matched layer," The Applied Computational Electromagnetic Society Journal, 2011.

[22] B. J. Frey, D. B. Leviton, and T. J. Madison, "Temperaturedependent refractive index of silicon and germanium," in Optomechanical Technologies for Astronomy, vol. 6273 of Proceedings of SPIE, Orlando, Fla, USA, May 2006. 

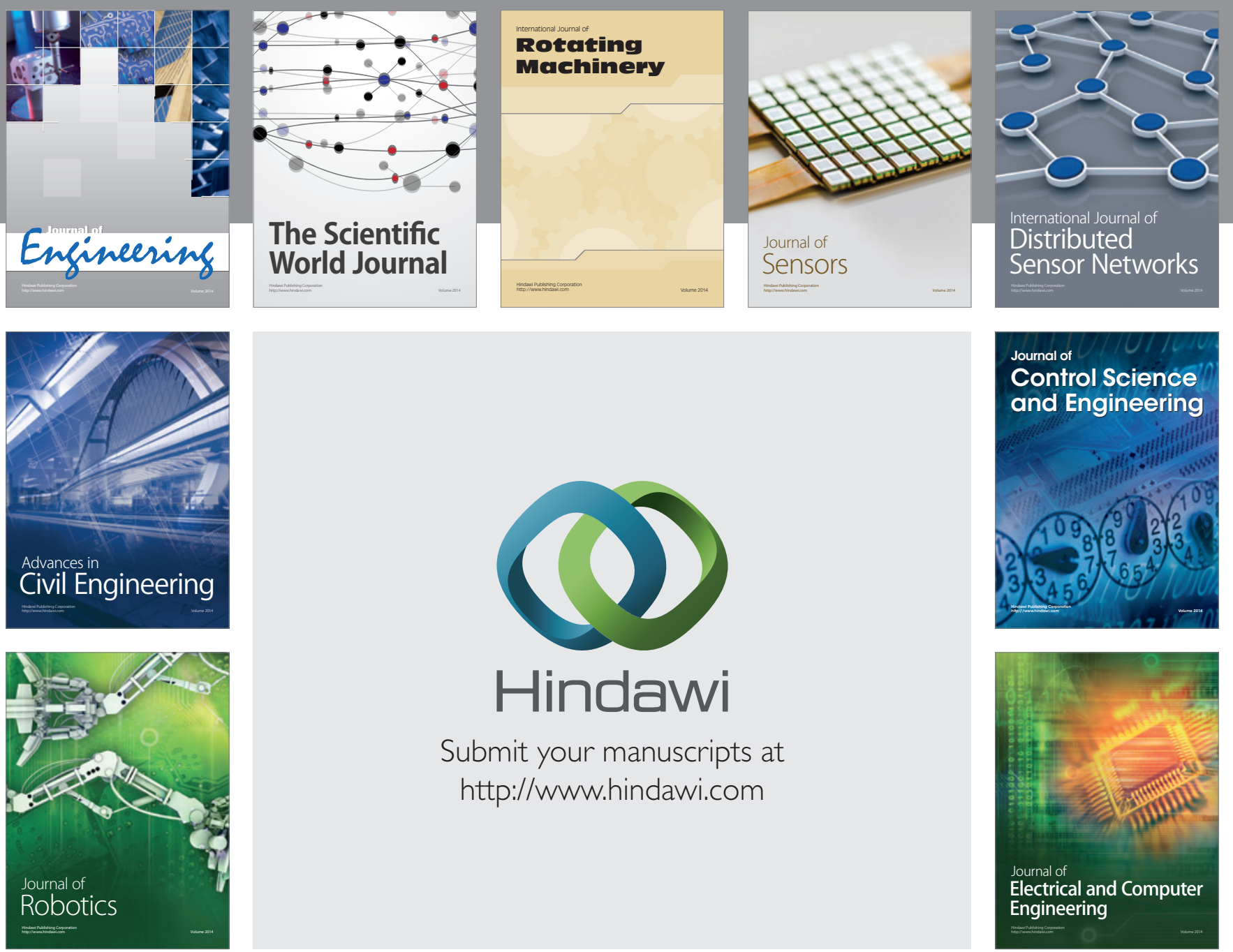

Submit your manuscripts at

http://www.hindawi.com
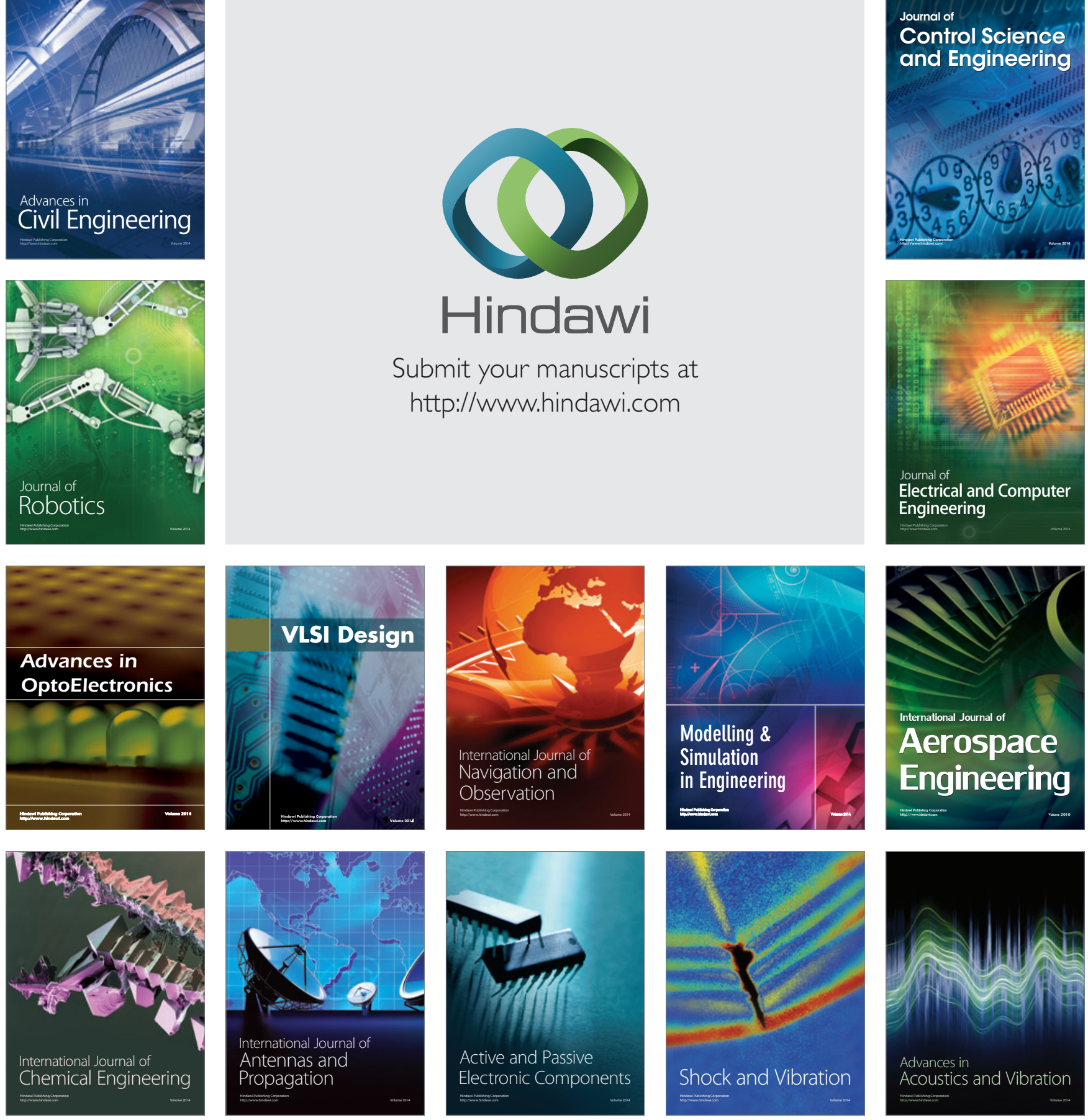